\title{
Fourth order elliptic operator-differential equations with unbounded operator boundary conditions in the Sobolev-type spaces
}

Eman S Al-Aidarous ${ }^{1}$, Araz R Aliev²,3*, Elvin S Rzayev ${ }^{3}$ and Hassan A Zedan ${ }^{4}$

\author{
"Correspondence: \\ alievaraz@yahoo.com \\ ${ }^{2}$ Baku State University, Baku, 1148, \\ Azerbaijan \\ ${ }^{3}$ Institute of Mathematics and \\ Mechanics of ANAS, Baku, 1141, \\ Azerbaijan \\ Full list of author information is \\ available at the end of the article
}

\begin{abstract}
Conditions for well-posed and unique solvability of a non-homogeneous boundary value problem for a class of fourth order elliptic operator-differential equations with an unbounded operator in boundary conditions are found in this work. Note that these solvability conditions are sufficient, and they are expressed only in terms of the properties of operator coefficients of the boundary value problem. Besides, the estimates for the norms of intermediate derivative operators in a Sobolev-type space are obtained, and their close relationship with the solvability conditions is established.

MSC: 34G10; 35J40; 47A50; 47D03

Keywords: elliptic operator-differential equations; unbounded operator boundary conditions; regular solution; regular solvability; the Sobolev-type space; intermediate derivative operators
\end{abstract}

\section{Introduction}

Many applied problems of mathematical physics require the study of spectral problems with a polynomial appearance of a parameter in the boundary conditions [1-3], while, in corresponding inverse problems, the unknown coefficients appearing in the equation and boundary conditions are found using known spectra [4-6]. However, some non-classical problems of mathematical physics [7] reduce to the problems with an operator in the boundary conditions. Note that the well-posed and unique solvability and Fredholmness of the boundary value problems for second and third order operator-differential equations with operator boundary conditions have been widely studied both on a finite interval and on the half-axis (see, e.g., [8-17] and the references therein, though far from being complete). The works dedicated to such kind of problems for fourth order operator-differential equations are relatively few. We can only mention $[18,19]$. Here it should be noted that the solvability of the boundary value problems for operator-differential equations of fourth and higher orders in case where the coefficients in the boundary conditions are only complex numbers has been extensively studied in [20-34]. See the references therein.

In this work, we treat the well-posed and unique solvability of a non-homogeneous boundary value problem for a fourth order elliptic operator-differential equation with un-

(c) 2015 Al-Aidarous et al. This article is distributed under the terms of the Creative Commons Attribution 4.0 International License (http://creativecommons.org/licenses/by/4.0/), which permits unrestricted use, distribution, and reproduction in any medium, provided you give appropriate credit to the original author(s) and the source, provide a link to the Creative Commons license, and indicate if changes were made. 
bounded operator boundary conditions. The solvability of this problem allows using the obtained abstract results in the study of both new classes of boundary value problems for fourth order elliptic partial differential equations and the corresponding spectral problems.

\section{Problem statement}

Let $H$ be a separable Hilbert space with scalar product $(x, y), x, y \in H$, and $A$ be a positive definite self-adjoint operator in $H\left(A=A^{*} \geq c E, c>0, E\right.$ is the identity operator). By $H_{\gamma}$ $(\gamma \geq 0)$ we will mean the scale of Hilbert spaces generated by the operator $A$, i.e., $H_{\gamma}=$ $D\left(A^{\gamma}\right),(x, y)_{\gamma}=\left(A^{\gamma} x, A^{\gamma} y\right), x, y \in D\left(A^{\gamma}\right)$, with $H_{0}=H,(x, y)_{0}=(x, y), x, y \in H$.

Denote by $L_{2}\left(\mathbb{R}_{+} ; H\right)$ the Hilbert space of all vector-valued functions defined on $\mathbb{R}_{+}=$ $(0,+\infty)$ with the values in $H$ and the finite norm

$$
\|f\|_{L_{2}\left(\mathbb{R}_{+} ; H\right)}=\left(\int_{0}^{+\infty}\|f(t)\|_{H}^{2} d t\right)^{1 / 2}
$$

Following [35], Chapter 1, we introduce the Hilbert space

$$
W_{2}^{4}\left(\mathbb{R}_{+} ; H\right)=\left\{u(t): u^{(4)}(t) \in L_{2}\left(\mathbb{R}_{+} ; H\right), A^{4} u(t) \in L_{2}\left(\mathbb{R}_{+} ; H\right)\right\}
$$

equipped with the norm

$$
\|u\|_{W_{2}^{4}\left(\mathbb{R}_{+} ; H\right)}=\left(\left\|u^{(4)}\right\|_{L_{2}\left(\mathbb{R}_{+} ; H\right)}^{2}+\left\|A^{4} u\right\|_{L_{2}\left(\mathbb{R}_{+} ; H\right)}^{2}\right)^{1 / 2}
$$

Hereinafter the derivatives $u^{(j)} \equiv \frac{d^{j} u}{d t^{j}}$ are understood in the sense of the theory of distributions in a Hilbert space [35]. Similarly we define the spaces $L_{2}(\mathbb{R} ; H)$ and $W_{2}^{4}(\mathbb{R} ; H)$, where $\mathbb{R}=(-\infty,+\infty)$.

By $L(X, Y)$ we mean the set of linear bounded operators acting from the Hilbert space $X$ to another Hilbert space $Y$. Fix some operator $K \in L\left(H_{5 / 2}, H_{3 / 2}\right)$. Let us define the following subspace of the space $W_{2}^{4}\left(\mathbb{R}_{+} ; H\right)$ :

$$
W_{2, K}^{4}\left(\mathbb{R}_{+} ; H\right)=\left\{u(t): u(t) \in W_{2}^{4}\left(\mathbb{R}_{+} ; H\right), u(0)=0, u^{\prime \prime}(0)=K u^{\prime}(0)\right\} .
$$

The trace theorem of [35], Chapter 1, implies the correctness of the last definition. Next, denote by $\sigma(\cdot)$ the spectrum of the operator $(\cdot)$.

Consider the following boundary value problem in the space $H$ :

$$
\begin{aligned}
& u^{(4)}(t)+A^{4} u(t)+\sum_{j=1}^{4} A_{j} u^{(4-j)}(t)=f(t), \quad t \in \mathbb{R}_{+}, \\
& u(0)=\varphi, \quad u^{\prime \prime}(0)-K u^{\prime}(0)=\psi,
\end{aligned}
$$

where $A=A^{*} \geq c E, c>0, A_{j}, j=1,2,3,4$, are linear and, in general, unbounded operators, $K \in L\left(H_{5 / 2}, H_{3 / 2}\right), f(t) \in L_{2}\left(\mathbb{R}_{+} ; H\right), \varphi \in H_{7 / 2}, \psi \in H_{3 / 2}, u(t) \in W_{2}^{4}\left(\mathbb{R}_{+} ; H\right)$.

Definition 2.1 If for every $f(t) \in L_{2}\left(\mathbb{R}_{+} ; H\right)$ and every $\varphi \in H_{7 / 2}, \psi \in H_{3 / 2}$ there exists a function $u(t) \in W_{2}^{4}\left(\mathbb{R}_{+} ; H\right)$ that satisfies equation (2.1) almost everywhere in $\mathbb{R}_{+}$, the 
boundary conditions (2.2) in the sense of convergence

$$
\begin{aligned}
& \lim _{t \rightarrow 0^{+}}\|u(t)-\varphi\|_{H_{7 / 2}}=0, \\
& \lim _{t \rightarrow 0^{+}}\left\|u^{\prime \prime}(t)-K u^{\prime}(t)-\psi\right\|_{H_{3 / 2}}=0
\end{aligned}
$$

and the estimate

$$
\|u\|_{W_{2}^{4}\left(\mathbb{R}_{+} ; H\right)} \leq \mathrm{const}\left(\|f\|_{L_{2}\left(\mathbb{R}_{+} ; H\right)}+\|\varphi\|_{H_{7 / 2}}+\|\psi\|_{H_{3 / 2}}\right)
$$

then the boundary value problem (2.1), (2.2) is said to be regularly solvable, and $u(t)$ is called a regular solution of the boundary value problem (2.1), (2.2).

The main purpose of this work is to find the conditions for regular solvability of the boundary value problem (2.1), (2.2) under some restrictions on its operator coefficients. To achieve this purpose, we use the estimates for the norms of intermediate derivative operators by the norm of an operator generated by the principal part of the considered equation and the given boundary conditions. This is precisely what distinguishes this paper. Note that the method offered in [23,36] and later developed in [31] to calculate the exact values of the norms of intermediate derivative operators is not directly applicable in our case. That is why we have to apply another procedure to estimate such norms, based on the classical inequalities of analysis. Being not too complicated and quite original, this procedure allows only upper estimates for the considered norms. But this is good enough for the purposes of this paper.

\section{Main results}

We first assume that $A_{j}=0, j=1,2,3,4$, and $\varphi=\psi=0$ in the boundary value problem (2.1), (2.2). Then we get a simpler boundary value problem

$$
\begin{aligned}
& u^{(4)}(t)+A^{4} u(t)=f(t), \quad t \in \mathbb{R}_{+} \\
& u(0)=0, \quad u^{\prime \prime}(0)=K u^{\prime}(0) .
\end{aligned}
$$

Denote by $P_{0}$ the operator that acts from $W_{2, K}^{4}\left(\mathbb{R}_{+} ; H\right)$ to $L_{2}\left(\mathbb{R}_{+} ; H\right)$ as follows:

$$
P_{0} u(t)=u^{(4)}(t)+A^{4} u(t), \quad u(t) \in W_{2, K}^{4}\left(\mathbb{R}_{+} ; H\right) .
$$

The following lemma is true.

Lemma 3.1 Let $B=A^{3 / 2} K A^{-5 / 2}$ and $-\sqrt{2} \notin \sigma(B)$. Then the equation $P_{0} u(t)=0$ has a unique zero solution in $W_{2, K}^{4}\left(\mathbb{R}_{+} ; H\right)$.

Proof Note that the general solution of the equation $P_{0} u(t)=0$ belonging to the space $W_{2}^{4}\left(\mathbb{R}_{+} ; H\right)$ has the following form:

$$
u_{0}(t)=e^{\omega_{1} t A} \xi_{0}+e^{\omega_{2} t A} \xi_{1}
$$


where the exponentials $e^{\omega_{1} t A}, e^{\omega_{2} t A}$ are the $\left(C_{0}\right)$ semigroups generated by $\omega_{1} A, \omega_{2} A$, respectively,

$$
\omega_{1}=-\frac{1}{\sqrt{2}}+\frac{1}{\sqrt{2}} i, \quad \omega_{2}=-\frac{1}{\sqrt{2}}-\frac{1}{\sqrt{2}} i,
$$

and $\xi_{0}, \xi_{1} \in H_{7 / 2}$. From (3.2) we have

$$
\left\{\begin{array}{l}
\xi_{0}+\xi_{1}=0, \\
\omega_{1}^{2} A^{2} \xi_{0}+\omega_{2}^{2} A^{2} \xi_{1}=K A\left(\omega_{1} \xi_{0}+\omega_{2} \xi_{1}\right) .
\end{array}\right.
$$

System (3.3) yields

$$
\begin{aligned}
& \xi_{1}=-\xi_{0}, \\
& \left(E+\frac{1}{\sqrt{2}} B\right) A^{7 / 2} \xi_{0}=0 .
\end{aligned}
$$

Then, by the condition $-\sqrt{2} \notin \sigma(B)$ of the lemma, it follows from equation (3.5) that $\xi_{0}=0$. Hence from (3.4) we have $\xi_{1}=0$. Consequently, $u_{0}(t)=0$. The lemma is proved.

The following theorem is true.

Theorem 3.2 Let $B=A^{3 / 2} K A^{-5 / 2}$ and $-\sqrt{2} \notin \sigma(B)$. Then, for every $f(t) \in L_{2}\left(\mathbb{R}_{+} ; H\right)$, the boundary value problem (3.1), (3.2) has a unique regular solution.

Proof By virtue of Lemma 3.1, the problem

$$
\begin{aligned}
& u^{(4)}(t)+A^{4} u(t)=0, \quad t \in \mathbb{R}_{+}, \\
& u(0)=0, \quad u^{\prime \prime}(0)=K u^{\prime}(0)
\end{aligned}
$$

has only zero solution in $W_{2, K}^{4}\left(\mathbb{R}_{+} ; H\right)$.

Let us show that the equation $P_{0} u(t)=f(t)$ has a solution $u(t) \in W_{2, K}^{4}\left(\mathbb{R}_{+} ; H\right)$ for every $f(t) \in L_{2}\left(\mathbb{R}_{+} ; H\right)$. For this aim, we first continue the vector function $f(t)$ by zero for $t<0$ and denote the obtained function by $F(t)$. Let $\hat{F}(\xi)$ be the Fourier transform of the vector function $F(t)$, i.e.,

$$
\hat{F}(\xi)=\frac{1}{\sqrt{2 \pi}} \int_{-\infty}^{+\infty} F(t) e^{-i \xi t} d t
$$

where the integral on the right-hand side is understood in the sense of mean convergence in $H$.

Applying direct and inverse Fourier transforms, we easily see that the vector function

$$
v(t)=\frac{1}{2 \pi} \int_{-\infty}^{+\infty}\left(\xi^{4} E+A^{4}\right)^{-1}\left(\int_{0}^{+\infty} f(s) e^{-i \xi s} d s\right) e^{i t \xi} d \xi, \quad t \in \mathbb{R},
$$

satisfies the equation

$$
v^{(4)}(t)+A^{4} v(t)=F(t)
$$


almost everywhere in $\mathbb{R}$. Let us show that $v(t) \in W_{2}^{4}(\mathbb{R} ; H)$. Let $\hat{v}(\xi)$ be the Fourier transform of the vector function $v(t)$. By the Plancherel theorem, we have

$$
\begin{aligned}
\|v(t)\|_{W_{2}^{4}(\mathbb{R} ; H)}^{2}= & \left\|v^{(4)}(t)\right\|_{L_{2}(\mathbb{R} ; H)}^{2}+\left\|A^{4} v(t)\right\|_{L_{2}(\mathbb{R} ; H)}^{2} \\
= & \left\|\xi^{4} \hat{v}(\xi)\right\|_{L_{2}(\mathbb{R} ; H)}^{2}+\left\|A^{4} \hat{v}(\xi)\right\|_{L_{2}(\mathbb{R} ; H)}^{2} \\
= & \left\|\xi^{4}\left(\xi^{4} E+A^{4}\right)^{-1} \hat{F}(\xi)\right\|_{L_{2}(\mathbb{R} ; H)}^{2} \\
& +\left\|A^{4}\left(\xi^{4} E+A^{4}\right)^{-1} \hat{F}(\xi)\right\|_{L_{2}(\mathbb{R} ; H)}^{2} \\
\leq & \sup _{\xi \in \mathbb{R}}\left\|\xi^{4}\left(\xi^{4} E+A^{4}\right)^{-1}\right\|_{H \rightarrow H}^{2}\|\hat{F}(\xi)\|_{L_{2}(\mathbb{R} ; H)}^{2} \\
& +\sup _{\xi \in \mathbb{R}}\left\|A^{4}\left(\xi^{4} E+A^{4}\right)^{-1}\right\|_{H \rightarrow H}^{2}\|\hat{F}(\xi)\|_{L_{2}(\mathbb{R} ; H)}^{2} \\
= & \left(\sup _{\xi \in \mathbb{R}}\left\|\xi^{4}\left(\xi^{4} E+A^{4}\right)^{-1}\right\|_{H \rightarrow H}^{2}+\sup _{\xi \in \mathbb{R}}\left\|A^{4}\left(\xi^{4} E+A^{4}\right)^{-1}\right\|_{H \rightarrow H}^{2}\right) \\
& \times\|F(t)\|_{L_{2}(\mathbb{R} ; H)}^{2} .
\end{aligned}
$$

According to the spectral theory of self-adjoint operators, for $\xi \in \mathbb{R}$, we have

$$
\begin{aligned}
& \left\|\xi^{4}\left(\xi^{4} E+A^{4}\right)^{-1}\right\| \leq \sup _{\sigma \in \sigma(A)}\left|\xi^{4}\left(\xi^{4}+\sigma^{4}\right)^{-1}\right| \leq 1, \\
& \left\|A^{4}\left(\xi^{4} E+A^{4}\right)^{-1}\right\| \leq \sup _{\sigma \in \sigma(A)}\left|\sigma^{4}\left(\xi^{4}+\sigma^{4}\right)^{-1}\right| \leq 1 .
\end{aligned}
$$

Then it follows from (3.6) that $v(t) \in W_{2}^{4}(\mathbb{R} ; H)$.

Now denote by $u_{1}(t)$ the restriction of the function $v(t)$ to $\mathbb{R}_{+}$. Then $u_{1}(t)$ belongs to the space $W_{2}^{4}\left(\mathbb{R}_{+} ; H\right)$, satisfies equation (3.1) almost everywhere in $\mathbb{R}_{+}$and, according to the trace theorem of [35], Chapter $1, u_{1}^{(j)}(0) \in H_{7 / 2-j}, j=0,1,2,3$.

Next, we seek the solution of the boundary value problem (3.1), (3.2) in the following form:

$$
u(t)=u_{1}(t)+e^{\omega_{1} t A} \eta_{0}+e^{\omega_{2} t A} \eta_{1}
$$

where

$$
\omega_{1}=-\frac{1}{\sqrt{2}}+\frac{1}{\sqrt{2}} i, \quad \omega_{2}=-\frac{1}{\sqrt{2}}-\frac{1}{\sqrt{2}} i
$$

and $\eta_{0}, \eta_{1} \in H_{7 / 2}$ are subject to be determined from (3.2). Then we get the following system:

$$
\left\{\begin{array}{l}
u_{1}(0)+\eta_{0}+\eta_{1}=0 \\
u_{1}^{\prime \prime}(0)+\omega_{1}^{2} A^{2} \eta_{0}+\omega_{2}^{2} A^{2} \eta_{1}=K\left(u_{1}^{\prime}(0)+\omega_{1} A \eta_{0}+\omega_{2} A \eta_{1}\right) .
\end{array}\right.
$$

Considering the relation

$$
\eta_{1}=-\eta_{0}-u_{1}(0)
$$


in the second equation of (3.7), and taking into account the condition $-\sqrt{2} \notin \sigma(B)$, we uniquely determine

$$
\eta_{0}=A^{-7 / 2}\left(E+\frac{1}{\sqrt{2}} B\right)^{-1} A^{7 / 2} \varsigma \in H_{7 / 2}
$$

where

$$
\varsigma=\frac{1}{2 i} A^{-2}\left[\omega_{2} K A u_{1}(0)-K u_{1}^{\prime}(0)+u_{1}^{\prime \prime}(0)-i A^{2} u_{1}(0)\right] \in H_{7 / 2} .
$$

Thus, $u(t)$ belongs to the space $W_{2}^{4}\left(\mathbb{R}_{+} ; H\right)$, satisfies equation (3.1) almost everywhere in $\mathbb{R}_{+}$and conditions (3.2).

On the other hand, the operator $P_{0}: W_{2, K}^{4}\left(\mathbb{R}_{+} ; H\right) \rightarrow L_{2}\left(\mathbb{R}_{+} ; H\right)$ is bounded:

$$
\left\|P_{0} u\right\|_{L_{2}\left(\mathbb{R}_{+} ; H\right)}^{2}=\left\|u^{(4)}+A^{4} u\right\|_{L_{2}\left(\mathbb{R}_{+} ; H\right)}^{2} \leq 2\|u\|_{W_{2}^{4}\left(\mathbb{R}_{+} ; H\right)}^{2} .
$$

Therefore, by the Banach inverse operator theorem, there exists the inverse operator $P_{0}^{-1}: L_{2}\left(\mathbb{R}_{+} ; H\right) \rightarrow W_{2, K}^{4}\left(\mathbb{R}_{+} ; H\right)$, and this operator is bounded. It follows

$$
\|u\|_{W_{2}^{4}\left(\mathbb{R}_{+} ; H\right)} \leq \text { const }\|f\|_{L_{2}\left(\mathbb{R}_{+} ; H\right)} .
$$

The theorem is proved.

In the sequel, we will need the following auxiliary statement.

Lemma 3.3 Let $B=A^{3 / 2} K A^{-5 / 2}$ and $\operatorname{Re} B \geq 0$. Then the inequality

$$
\left\|P_{0} u\right\|_{L_{2}\left(\mathbb{R}_{+} ; H\right)}^{2} \geq\|u\|_{W_{2}^{4}\left(\mathbb{R}_{+} ; H\right)}^{2}+2\left\|A^{2} u^{\prime \prime}\right\|_{L_{2}\left(\mathbb{R}_{+} ; H\right)}^{2}
$$

is true for every $u(t) \in W_{2, K}^{4}\left(\mathbb{R}_{+} ; H\right)$.

Proof Integrating by parts, for $u(t) \in W_{2, K}^{4}\left(\mathbb{R}_{+} ; H\right)$, we obtain

$$
\operatorname{Re}\left(u^{(4)}, A^{4} u\right)_{L_{2}\left(\mathbb{R}_{+} ; H\right)}=\operatorname{Re}\left(B A^{5 / 2} u^{\prime}(0), A^{5 / 2} u^{\prime}(0)\right)+\left\|A^{2} u^{\prime \prime}\right\|_{L_{2}\left(\mathbb{R}_{+} ; H\right)}^{2}
$$

Due to (3.10), we have

$$
\begin{aligned}
\left\|P_{0} u\right\|_{L_{2}\left(\mathbb{R}_{+} ; H\right)}^{2} & =\left\|u^{(4)}\right\|_{L_{2}\left(\mathbb{R}_{+} ; H\right)}^{2}+\left\|A^{4} u\right\|_{L_{2}\left(\mathbb{R}_{+} ; H\right)}^{2}+2 \operatorname{Re}\left(u^{(4)}, A^{4} u\right)_{L_{2}\left(\mathbb{R}_{+} ; H\right)} \\
& =\|u\|_{W_{2}^{4}\left(\mathbb{R}_{+} ; H\right)}^{2}+2 \operatorname{Re}\left(B A^{5 / 2} u^{\prime}(0), A^{5 / 2} u^{\prime}(0)\right)+2\left\|A^{2} u^{\prime \prime}\right\|_{L_{2}\left(\mathbb{R}_{+} ; H\right)}^{2} .
\end{aligned}
$$

As $\operatorname{Re} B \geq 0$, equality (3.11) implies the validity of inequality (3.9). The lemma is proved.

Note that Theorem 3.2 combined with Lemma 3.1 implies that the operator $P_{0}$, under the condition $-\sqrt{2} \notin \sigma(B)$ with $B=A^{3 / 2} K A^{-5 / 2}$, maps the space $W_{2, K}^{4}\left(\mathbb{R}_{+} ; H\right)$ isomorphically onto $L_{2}\left(\mathbb{R}_{+} ; H\right)$. Consequently, the norm $\left\|P_{0} u\right\|_{L_{2}\left(\mathbb{R}_{+} ; H\right)}$ is equivalent in $W_{2, K}^{4}\left(\mathbb{R}_{+} ; H\right)$ to the initial norm $\|u\|_{W_{2}^{4}\left(\mathbb{R}_{+} ; H\right)}$. And, as the intermediate derivative operators

$$
A^{j} \frac{d^{4-j}}{d t^{4-j}}: W_{2, K}^{4}\left(\mathbb{R}_{+} ; H\right) \rightarrow L_{2}\left(\mathbb{R}_{+} ; H\right), \quad j=1,2,3,4,
$$


are continuous (see [35]), the norms of these operators can be estimated through the norm $\left\|P_{0} u\right\|_{L_{2}\left(\mathbb{R}_{+} ; H\right)}$. The need for these estimates arises when one tries to establish solvability conditions for the boundary value problem (2.1), (2.2) using only the properties of its operator coefficients.

Theorem 3.4 Let $B=A^{3 / 2} K A^{-5 / 2}$ and $\operatorname{Re} B \geq 0$. Then the estimates

$$
\left\|A^{j} u^{(4-j)}\right\|_{L_{2}\left(\mathbb{R}_{+} ; H\right)} \leq c_{j}\left\|P_{0} u\right\|_{L_{2}\left(\mathbb{R}_{+} ; H\right)}, \quad j=0,1,2,3,4
$$

are true for every $u(t) \in W_{2, K}^{4}\left(\mathbb{R}_{+} ; H\right)$ with

$$
c_{0}=c_{1}=c_{4}=1, \quad c_{2}=\frac{1}{2}, \quad c_{3}=\frac{1}{\sqrt{2}} .
$$

Proof We first multiply both sides of equation (3.1) scalarly by $A^{4} u(t)$ in the space $L_{2}\left(\mathbb{R}_{+} ; H\right)$ and then integrate by parts. Then, taking into account (3.10) and the condition $\operatorname{Re} B \geq 0$, we have

$$
\begin{aligned}
& \operatorname{Re}\left(P_{0} u, A^{4} u\right)_{L_{2}\left(\mathbb{R}_{+} ; H\right)} \\
& \quad=\operatorname{Re}\left(u^{(4)}+A^{4} u, A^{4} u\right)_{L_{2}\left(\mathbb{R}_{+} ; H\right)} \\
& \quad=\left\|A^{4} u\right\|_{L_{2}\left(\mathbb{R}_{+} ; H\right)}^{2}+\operatorname{Re}\left(B A^{5 / 2} u^{\prime}(0), A^{5 / 2} u^{\prime}(0)\right)+\left\|A^{2} u^{\prime \prime}\right\|_{L_{2}\left(\mathbb{R}_{+} ; H\right)}^{2} \\
& \quad \geq\left\|A^{4} u\right\|_{L_{2}\left(\mathbb{R}_{+} ; H\right)}^{2}+\left\|A^{2} u^{\prime \prime}\right\|_{L_{2}\left(\mathbb{R}_{+} ; H\right)}^{2} .
\end{aligned}
$$

Applying the Cauchy-Schwarz inequality and then the Young inequality to the left-hand side of (3.13), we get

$$
\begin{aligned}
& \left\|A^{4} u\right\|_{L_{2}\left(\mathbb{R}_{+} ; H\right)}^{2}+\left\|A^{2} u^{\prime \prime}\right\|_{L_{2}\left(\mathbb{R}_{+} ; H\right)}^{2} \\
& \quad \leq\left\|P_{0} u\right\|_{L_{2}\left(\mathbb{R}_{+} ; H\right)}\left\|A^{4} u\right\|_{L_{2}\left(\mathbb{R}_{+} ; H\right)} \\
& \quad \leq \frac{\delta}{2}\left\|P_{0} u\right\|_{L_{2}\left(\mathbb{R}_{+} ; H\right)}^{2}+\frac{1}{2 \delta}\left\|A^{4} u\right\|_{L_{2}\left(\mathbb{R}_{+} ; H\right)}^{2}, \quad \delta>0 .
\end{aligned}
$$

Assume $\delta=\frac{1}{2}$ in (3.14). Then we obtain

$$
\left\|A^{2} u^{\prime \prime}\right\|_{L_{2}\left(\mathbb{R}_{+} ; H\right)}^{2} \leq \frac{1}{4}\left\|P_{0} u\right\|_{L_{2}\left(\mathbb{R}_{+} ; H\right)}^{2}
$$

or

$$
\left\|A^{2} u^{\prime \prime}\right\|_{L_{2}\left(\mathbb{R}_{+} ; H\right)} \leq \frac{1}{2}\left\|P_{0} u\right\|_{L_{2}\left(\mathbb{R}_{+} ; H\right)} .
$$

On the other hand, from (3.14) we have

$$
\left\|A^{4} u\right\|_{L_{2}\left(\mathbb{R}_{+} ; H\right)}^{2} \leq\left\|P_{0} u\right\|_{L_{2}\left(\mathbb{R}_{+} ; H\right)}\left\|A^{4} u\right\|_{L_{2}\left(\mathbb{R}_{+} ; H\right)} .
$$

Therefore,

$$
\left\|A^{4} u\right\|_{L_{2}\left(\mathbb{R}_{+} ; H\right)} \leq\left\|P_{0} u\right\|_{L_{2}\left(\mathbb{R}_{+} ; H\right)} .
$$


Note that the validity of (3.16) can be also obtained from inequality (3.9). Besides, inequality (3.9) implies the validity of the following one:

$$
\left\|u^{(4)}\right\|_{L_{2}\left(\mathbb{R}_{+} ; H\right)} \leq\left\|P_{0} u\right\|_{L_{2}\left(\mathbb{R}_{+} ; H\right)}
$$

Now let us estimate the norm $\left\|A^{3} u^{\prime}\right\|_{L_{2}\left(\mathbb{R}_{+} ; H\right)}$. Integrating by parts, taking into account $u(t) \in W_{2, K}^{4}\left(\mathbb{R}_{+} ; H\right)$, and then applying the Cauchy-Schwarz inequality and inequalities (3.15), (3.16), we obtain

$$
\begin{aligned}
\left\|A^{3} u^{\prime}\right\|_{L_{2}\left(\mathbb{R}_{+} ; H\right)}^{2} & =\int_{0}^{+\infty}\left(A^{3} u^{\prime}, A^{3} u^{\prime}\right) d t \\
& =\left.\left(A^{3} u, A^{3} u^{\prime}\right)\right|_{0} ^{+\infty}-\int_{0}^{+\infty}\left(A^{4} u, A^{2} u^{\prime \prime}\right) d t \\
& =-\int_{0}^{+\infty}\left(A^{4} u, A^{2} u^{\prime \prime}\right) d t \leq\left\|A^{4} u\right\|_{L_{2}\left(\mathbb{R}_{+} ; H\right)}\left\|A^{2} u^{\prime \prime}\right\|_{L_{2}\left(\mathbb{R}_{+} ; H\right)} \\
& \leq \frac{1}{2}\left\|P_{0} u\right\|_{L_{2}\left(\mathbb{R}_{+} ; H\right)}^{2} .
\end{aligned}
$$

Consequently,

$$
\left\|A^{3} u^{\prime}\right\|_{L_{2}\left(\mathbb{R}_{+} ; H\right)} \leq \frac{1}{\sqrt{2}}\left\|P_{0} u\right\|_{L_{2}\left(\mathbb{R}_{+} ; H\right)} .
$$

Finally, we proceed to estimate the norm $\left\|A u^{\prime \prime \prime}\right\|_{L_{2}\left(\mathbb{R}_{+} ; H\right)}$. It was shown in [25] that the inequality

$$
\left\|A u^{\prime \prime \prime}\right\|_{L_{2}\left(\mathbb{R}_{+} ; H\right)}^{2} \leq 2\left\|A^{2} u^{\prime \prime}\right\|_{L_{2}\left(\mathbb{R}_{+} ; H\right)}\left\|u^{(4)}\right\|_{L_{2}\left(\mathbb{R}_{+} ; H\right)}
$$

is true for $u(t) \in W_{2}^{4}\left(\mathbb{R}_{+} ; H\right)$. Considering inequalities (3.15) and (3.17) in (3.18), we have

$$
\left\|A u^{\prime \prime \prime}\right\|_{L_{2}\left(\mathbb{R}_{+} ; H\right)}^{2} \leq\left\|P_{0} u\right\|_{L_{2}\left(\mathbb{R}_{+} ; H\right)}^{2}
$$

or

$$
\left\|A u^{\prime \prime \prime}\right\|_{L_{2}\left(\mathbb{R}_{+} ; H\right)} \leq\left\|P_{0} u\right\|_{L_{2}\left(\mathbb{R}_{+} ; H\right)} .
$$

The theorem is proved.

Now we consider another case of problem (2.1), (2.2) with $A_{j} \neq 0, j=1,2,3,4$, while $\varphi$ and $\psi$ are equal to zero as before:

$$
\begin{aligned}
& u^{(4)}(t)+A^{4} u(t)+\sum_{j=1}^{4} A_{j} u^{(4-j)}(t)=f(t), \quad t \in \mathbb{R}_{+}, \\
& u(0)=0, \quad u^{\prime \prime}(0)=K u^{\prime}(0) .
\end{aligned}
$$

Denote by $P$ the operator that acts from $W_{2, K}^{4}\left(\mathbb{R}_{+} ; H\right)$ to $L_{2}\left(\mathbb{R}_{+} ; H\right)$ as follows:

$$
P u(t)=u^{(4)}(t)+A^{4} u(t)+\sum_{j=1}^{4} A_{j} u^{(4-j)}(t), \quad u(t) \in W_{2, K}^{4}\left(\mathbb{R}_{+} ; H\right) .
$$


The following lemma is true.

Lemma 3.5 Let $A_{j} A^{-j} \in L(H, H), j=1,2,3,4$. Then the operator $P$ is a bounded operator from $W_{2, K}^{4}\left(\mathbb{R}_{+} ; H\right)$ to $L_{2}\left(\mathbb{R}_{+} ; H\right)$.

Proof Taking into account the conditions of the lemma and inequality (3.8), for every $u(t) \in W_{2, K}^{4}\left(\mathbb{R}_{+} ; H\right)$, we have

$$
\begin{aligned}
\|P u\|_{L_{2}\left(\mathbb{R}_{+} ; H\right)} & \leq\left\|P_{0} u\right\|_{L_{2}\left(\mathbb{R}_{+} ; H\right)}+\left\|\sum_{j=1}^{4} A_{j} u^{(4-j)}\right\|_{L_{2}\left(\mathbb{R}_{+} ; H\right)} \\
& \leq \sqrt{2}\|u\|_{W_{2}^{4}\left(\mathbb{R}_{+} ; H\right)}+\sum_{j=1}^{4}\left\|A_{j} u^{(4-j)}\right\|_{L_{2}\left(\mathbb{R}_{+} ; H\right)} \\
& \leq \sqrt{2}\|u\|_{W_{2}^{4}\left(\mathbb{R}_{+} ; H\right)}+\sum_{j=1}^{4}\left\|A_{j} A^{-j}\right\|_{H \rightarrow H}\left\|A^{j} u^{(4-j)}\right\|_{L_{2}\left(\mathbb{R}_{+} ; H\right)} .
\end{aligned}
$$

Then, by the theorem on intermediate derivatives [35], Chapter 1, from (3.21) we obtain

$$
\|P u\|_{L_{2}\left(\mathbb{R}_{+} ; H\right)} \leq \text { const }\|u\|_{W_{2}^{4}\left(\mathbb{R}_{+} ; H\right)} .
$$

The lemma is proved.

The following theorem on solvability of the boundary value problem (3.19), (3.20) is true, stated only in terms of the properties of its operator coefficients.

Theorem 3.6 Let $B=A^{3 / 2} K A^{-5 / 2}, \operatorname{Re} B \geq 0, A_{j} A^{-j} \in L(H, H), j=1,2,3,4$, and the inequality

$$
\alpha=\sum_{j=1}^{4} c_{j}\left\|A_{j} A^{-j}\right\|_{H \rightarrow H}<1
$$

be fulfilled, where the numbers $c_{j}, j=1,2,3,4$, are defined in Theorem 3.4, i.e.,

$$
c_{1}=c_{4}=1, \quad c_{2}=\frac{1}{2}, \quad c_{3}=\frac{1}{\sqrt{2}} .
$$

Then the boundary value problem (3.19), (3.20) has a unique regular solution for every $f(t) \in L_{2}\left(\mathbb{R}_{+} ; H\right)$.

Proof First we rewrite the boundary value problem (3.19), (3.20) in the form of operator equation

$$
P_{0} u(t)+\left(P-P_{0}\right) u(t)=f(t),
$$

where $f(t) \in L_{2}\left(\mathbb{R}_{+} ; H\right), u(t) \in W_{2, K}^{4}\left(\mathbb{R}_{+} ; H\right)$. 
Note that the conditions $B=A^{3 / 2} K A^{-5 / 2}, \operatorname{Re} B \geq 0$ provide the existence of the bounded inverse operator $P_{0}^{-1}$ from $L_{2}\left(\mathbb{R}_{+} ; H\right)$ to $W_{2, K}^{4}\left(\mathbb{R}_{+} ; H\right)$. Replacing $u(t)=P_{0}^{-1} v(t)$, where $v(t) \in$ $L_{2}\left(\mathbb{R}_{+} ; H\right)$, we arrive at the following equation in the space $L_{2}\left(\mathbb{R}_{+} ; H\right)$ :

$$
v(t)+\left(P-P_{0}\right) P_{0}^{-1} v(t)=f(t) .
$$

In this case, taking into account estimates (3.12), for every $v(t) \in L_{2}\left(\mathbb{R}_{+} ; H\right)$, we obtain

$$
\begin{aligned}
\left\|\left(P-P_{0}\right) P_{0}^{-1} v\right\|_{L_{2}\left(\mathbb{R}_{+} ; H\right)} & =\left\|\left(P-P_{0}\right) u\right\|_{L_{2}\left(\mathbb{R}_{+} ; H\right)} \\
& \leq \sum_{j=1}^{4}\left\|A_{j} A^{-j}\right\|_{H \rightarrow H}\left\|A^{j} u^{(4-j)}\right\|_{L_{2}\left(\mathbb{R}_{+} ; H\right)} \\
& \leq \sum_{j=1}^{4} c_{j}\left\|A_{j} A^{-j}\right\|_{H \rightarrow H}\left\|P_{0} u\right\|_{L_{2}\left(\mathbb{R}_{+} ; H\right)} \\
& =\alpha\|v\|_{L_{2}\left(\mathbb{R}_{+} ; H\right)} .
\end{aligned}
$$

As $\alpha<1$, the operator $E+\left(P-P_{0}\right) P_{0}^{-1}$ has an inverse in the space $L_{2}\left(\mathbb{R}_{+} ; H\right)$. Consequently, equation (3.22) is uniquely solvable in the space $W_{2, K}^{4}\left(\mathbb{R}_{+} ; H\right)$, and

$$
u(t)=P_{0}^{-1}\left(E+\left(P-P_{0}\right) P_{0}^{-1}\right)^{-1} f(t)
$$

It follows

$$
\begin{aligned}
& \|u\|_{W_{2}^{4}\left(\mathbb{R}_{+} ; H\right)} \\
& \quad \leq\left\|P_{0}^{-1}\right\|_{L_{2}\left(\mathbb{R}_{+} ; H\right) \rightarrow W_{2}^{4}\left(\mathbb{R}_{+} ; H\right)}\left\|\left(E+\left(P-P_{0}\right) P_{0}^{-1}\right)^{-1}\right\|_{L_{2}\left(\mathbb{R}_{+} ; H\right) \rightarrow L_{2}\left(\mathbb{R}_{+} ; H\right)}\|f\|_{L_{2}\left(\mathbb{R}_{+} ; H\right)} \\
& \quad \leq \text { const }\|f\|_{L_{2}\left(\mathbb{R}_{+} ; H\right)} .
\end{aligned}
$$

The theorem is proved.

Remark 3.7 In Theorem 3.6, the condition $\operatorname{Re} B \geq 0$ with $B=A^{3 / 2} K A^{-5 / 2}$ allows to omit the condition $-\sqrt{2} \notin \sigma(B)$.

Finally, from Theorem 3.6 we can obtain the conditions for the regular solvability of the boundary value problem (2.1), (2.2).

Theorem 3.8 Let all the conditions of Theorem 3.6 be fulfilled. Then the boundary value problem (2.1), (2.2) is regularly solvable.

Proof Obviously, in case $\varphi=\psi=0$ the regular solvability of the boundary value problem (2.1), (2.2) follows from Theorem 3.6.

In case $A_{j}=0, j=1,2,3,4$, and $f(t)=0$, we have the problem

$$
\begin{aligned}
& u^{(4)}(t)+A^{4} u(t)=0, \quad t \in \mathbb{R}_{+}, \\
& u(0)=\varphi, \quad u^{\prime \prime}(0)-K u^{\prime}(0)=\psi,
\end{aligned}
$$


where $\varphi \in H_{7 / 2}, \psi \in H_{3 / 2}$. The solution of problem (3.23), (3.24) will be sought in the following form:

$$
u_{0}(t)=e^{\omega_{1} t A} \zeta_{0}+e^{\omega_{2} t A} \zeta_{1}
$$

where

$$
\omega_{1}=-\frac{1}{\sqrt{2}}+\frac{1}{\sqrt{2}} i, \quad \omega_{2}=-\frac{1}{\sqrt{2}}-\frac{1}{\sqrt{2}} i,
$$

and $\zeta_{0}, \zeta_{1}$ are the unknown vectors to be determined from conditions (3.24):

$$
\left\{\begin{array}{l}
\zeta_{0}+\zeta_{1}=\varphi \\
\omega_{1}^{2} A^{2} \zeta_{0}+\omega_{2}^{2} A^{2} \zeta_{1}-K A\left(\omega_{1} \zeta_{0}+\omega_{2} \zeta_{1}\right)=\psi
\end{array}\right.
$$

Considering the relation

$$
\zeta_{1}=\varphi-\zeta_{0}
$$

in the second equation of system (3.26) and taking into account the condition $-\sqrt{2} \notin \sigma(B)$, we uniquely determine

$$
\zeta_{0}=\frac{i}{2} A^{-7 / 2}\left(E+\frac{1}{\sqrt{2}} B\right)^{-1} A^{3 / 2}\left(\psi-\omega_{2}^{2} A^{2} \varphi+\omega_{2} K A \varphi\right) .
$$

Then

$$
\zeta_{1}=\varphi-\frac{i}{2} A^{-7 / 2}\left(E+\frac{1}{\sqrt{2}} B\right)^{-1} A^{3 / 2}\left(\psi-\omega_{2}^{2} A^{2} \varphi+\omega_{2} K A \varphi\right) .
$$

It is clear that $\zeta_{0}, \zeta_{1} \in H_{7 / 2}$. Consequently, from the representation (3.25) we have [35], Chapter 1

$$
\begin{aligned}
\left\|u_{0}\right\|_{W_{2}^{4}\left(\mathbb{R}_{+} ; H\right)} & \leq \operatorname{const}\left(\left\|\zeta_{0}\right\|_{H_{7 / 2}}+\left\|\zeta_{1}\right\|_{H_{7 / 2}}\right) \\
& \leq \operatorname{const}\left(\|\varphi\|_{H_{7 / 2}}+\|\psi\|_{H_{3 / 2}}\right) .
\end{aligned}
$$

Now we can directly proceed to the study of boundary value problem (2.1), (2.2). We will seek the solution of the boundary value problem (2.1), (2.2) in the form $u(t)=v(t)+u_{0}(t)$, where $u_{0}(t)$ is a regular solution of problem (3.23), (3.24). Then the function $v(t)$ is the solution of the boundary value problem

$$
\begin{aligned}
& v^{(4)}(t)+A^{4} v(t)+\sum_{j=1}^{4} A_{j} v^{(4-j)}(t)=g(t), \quad t \in \mathbb{R}_{+}, \\
& v(0)=0, \quad v^{\prime \prime}(0)=K v^{\prime}(0),
\end{aligned}
$$

where

$$
g(t) \equiv-\sum_{j=1}^{4} A_{j} u_{0}^{(4-j)}(t)+f(t) .
$$


Estimating the norm of the function $g(t)$ in $L_{2}\left(\mathbb{R}_{+} ; H\right)$, we obtain

$$
\begin{aligned}
\|g\|_{L_{2}\left(\mathbb{R}_{+} ; H\right)} & \leq\left\|\sum_{j=1}^{4} A_{j} u_{0}^{(4-j)}\right\|_{L_{2}\left(\mathbb{R}_{+} ; H\right)}+\|f\|_{L_{2}\left(\mathbb{R}_{+} ; H\right)} \\
& \leq \sum_{j=1}^{4}\left\|A_{j} A^{-j}\right\|\left\|A^{j} u_{0}^{(4-j)}\right\|_{L_{2}\left(\mathbb{R}_{+} ; H\right)}+\|f\|_{L_{2}\left(\mathbb{R}_{+} ; H\right)} \\
& \leq \operatorname{const}\left(\|\varphi\|_{H_{7 / 2}}+\|\psi\|_{H_{3 / 2}}+\|f\|_{L_{2}\left(\mathbb{R}_{+} ; H\right)}\right) .
\end{aligned}
$$

Consequently, by Theorem 3.6 and estimate (3.27), we have

$$
\begin{aligned}
\|u\|_{W_{2}^{4}\left(\mathbb{R}_{+} ; H\right)} & \leq\|v\|_{W_{2}^{4}\left(\mathbb{R}_{+} ; H\right)}+\left\|u_{0}\right\|_{W_{2}^{4}\left(\mathbb{R}_{+} ; H\right)} \\
& \leq \text { const }\|g\|_{L_{2}\left(\mathbb{R}_{+} ; H\right)}+\left\|u_{0}\right\|_{W_{2}^{4}\left(\mathbb{R}_{+} ; H\right)} \\
& \leq \operatorname{const}\left(\|\varphi\|_{H_{7 / 2}}+\|\psi\|_{H_{3 / 2}}+\|f\|_{L_{2}\left(\mathbb{R}_{+} ; H\right)}\right) .
\end{aligned}
$$

The theorem is proved.

Note that the case where the operator $\operatorname{Re} B$ is not non-negative requires special consideration.

\section{Appendix}

Now we apply the abstract results of Theorem 3.6 to a problem for partial differential equations.

On the half-strip $\mathbb{R}_{+} \times(0, \pi)$, consider the following boundary value problem for fourth order elliptic equations:

$$
\begin{aligned}
& \frac{\partial^{4} u(t, x)}{\partial t^{4}}+\frac{\partial^{4} u(t, x)}{\partial x^{4}}+\sum_{j=1}^{4} p_{j}(x) \frac{\partial^{4} u(t, x)}{\partial t^{4-j} \partial x^{j}}=f(t, x) \\
& u(0, x)=0, \quad \frac{\partial^{2} u(0, x)}{\partial t^{2}}=a \frac{\partial^{2} u(0, x)}{\partial t \partial x} \\
& \frac{\partial^{2 k} u(t, 0)}{\partial x^{2 k}}=\frac{\partial^{2 k} u(t, \pi)}{\partial x^{2 k}}=0, \quad k=0,1
\end{aligned}
$$

where $a$ is a complex number, $p_{j}(x), j=1,2,3,4$, are bounded functions on the interval $(0, \pi), f(t, x) \in L_{2}\left(\mathbb{R}_{+} ; L_{2}(0, \pi)\right)$. Note that this problem can be reduced to the boundary value problem (3.19), (3.20). Here $H=L_{2}(0, \pi), A_{j}=p_{j}(x) \frac{\partial^{j}}{\partial x^{j}}, j=1,2,3,4$. The operator $A^{4}$ is defined in $L_{2}(0, \pi)$ by the equality $A^{4} u=\frac{d^{4} u}{d x^{4}}$ with the conditions $u(0)=u(\pi)=u^{\prime \prime}(0)=$ $u^{\prime \prime}(\pi)=0$, and the operator $K$ is defined in $L_{2}(0, \pi)$ by the equality $K u=a \frac{d u}{d x}$.

Taking into account Theorem 3.6, we obtain that under the conditions $\operatorname{Re} a \geq 0$ and $\sum_{j=1}^{4} c_{j} \sup _{0 \leq x \leq \pi}\left|p_{j}(x)\right|<1$, the boundary value problem (A.1)-(A.3) has a unique solution in the space $W_{2}^{4}\left(\mathbb{R}_{+} ; L_{2}(0, \pi)\right)$. 
Authors' contributions

The authors contributed equally to this work. All authors read and approved the final manuscript.

\section{Author details}

${ }^{1}$ King Abdulaziz University, Jeddah, 21589, Saudi Arabia. ${ }^{2}$ Baku State University, Baku, 1148, Azerbaijan. ${ }^{3}$ Institute of Mathematics and Mechanics of ANAS, Baku, 1141, Azerbaijan. ${ }^{4}$ Kafr El-Sheikh University, Kafr El-Sheikh, 33516, Egypt.

\section{Acknowledgements}

This work was supported by the Deanship of Scientific Research (DSR), King Abdulaziz University, Jeddah, under grant No. (363-686-D1435). The authors, therefore, gratefully acknowledge the DSR technical and financial support.

Received: 10 June 2015 Accepted: 9 October 2015 Published online: 22 October 2015

\section{References}

1. Shkalikov, AA: Boundary value problems for ordinary differential equations with a parameter in the boundary conditions. Tr. Semin. Petrovsk. 9, 190-229 (1983) (in Russian)

2. Akhtyamov, AM: Calculation of the coefficients of expansions in derivative chains of a spectral problem. Math. Notes 51(6), 618-619 (1992) (translated from Mat. Zametki 51(6), 137-139 (1992))

3. Akhtyamov, AM: On coefficients of eigenfunction expansions for boundary-value problems with parameter in boundary conditions. Math. Notes 75(4), 462-474 (2004) (translated from Mat. Zametki 75(4), 493-506 (2004))

4. Nabiev, IM, Shukurov, AS: Properties of the spectrum and uniqueness of reconstruction of Sturm-Liouville operator with a spectral parameter in the boundary condition. Proc. Inst. Math. Mech. Natl. Acad. Sci. Azerb. 40, 332-341 (2014) (special issue)

5. Mamedov, KR, Cetinkaya, FA: Inverse problem for a class of Sturm-Liouville operator with spectral parameter in boundary condition. Bound. Value Probl. 2013, 183 (2013)

6. Sadovnichii, VA, Sultanaev, YT, Akhtyamov, AM: Inverse problem for an operator pencil with nonseparated boundary conditions. Dokl. Math. 79(2), 169-171 (2009) (translated from Dokl. Akad. Nauk 425(1), 31-33 (2009))

7. Bitsadze, AV: Some Classes of Partial Differential Equations. Nauka, Moscow (1981) (in Russian)

8. Gasymov, MG, Mirzoev, SS: Solvability of boundary value problems for second-order operator-differential equations of elliptic type. Differ. Equ. 28(4), 528-536 (1992) (translated from Differ. Uravn. 28(4), 651-661 (1992))

9. Mirzoev, SS, Yagubova, KV: On the solvability of boundary value problems with operators in the boundary conditions for a class of second-order operator-differential equations. Dokl. Nats. Akad. Nauk Azerb. 57(1-3), 12-17 (2001) (in Russian)

10. Aliev, AR, Babayeva, SF: On the boundary value problem with the operator in boundary conditions for the operator-differential equation of the third order. J. Math. Phys. Anal. Geom. 6(4), 347-361 (2010)

11. Aliev, BA: A boundary value problem for a second order elliptic differential-operator equation with a spectral parameter and operator boundary conditions. Proc. Inst. Math. Mech. Natl. Acad. Sci. Azerb. 32(40), 21-46 (2010)

12. Aliev, BA, Yakubov, Y: Second order elliptic differential-operator equations with unbounded operator boundary conditions in UMD Banach spaces. Integral Equ. Oper. Theory 69(2), 269-300 (2011)

13. Mirzoev, SS, Salimov, MY: On the solvability of the boundary-value problem for second-order equations in Hilbert space with an operator coefficient in the boundary condition. Math. Notes 91(5-6), 808-815 (2012) (translated from Mat. Zametki 91(6), 861-869 (2012))

14. Mirzoev, SS, Aliev, AR, Rustamova, LA: Solvability conditions for boundary-value problems for elliptic operator-differential equations with discontinuous coefficient. Math. Notes 92(5-6), 722-726 (2012) (translated from Mat. Zametki 92(5), 789-793 (2012))

15. Mirzoev, SS, Aliev, AR, Rustamova, LA: On the boundary value problem with the operator in boundary conditions for the operator-differential equation of second order with discontinuous coefficients. J. Math. Phys. Anal. Geom. 9(2), 207-226 (2013)

16. Aliev, AR, Muradova, NL: Third-order operator-differential equations with discontinuous coefficients and operators in the boundary conditions. Electron. J. Differ. Equ. 2013, 219 (2013)

17. Gasimova, GM: On solvability conditions of a boundary value problem with an operator in the boundary condition for a second order elliptic operator-differential equation. Proc. Inst. Math. Mech. Natl. Acad. Sci. Azerb. 40, 172-177 (2014) (special issue)

18. Aliev, AR, Rzayev, ES: Solvability of boundary value problem for elliptic operator-differential equations of fourth order with operator boundary conditions. Proc. Inst. Math. Mech. Natl. Acad. Sci. Azerb. 40 13-22 (2014) (special issue)

19. Aliev, BA, Yakubov, Y: Fredholm property of boundary value problems for a fourth-order elliptic differential-operator equation with operator boundary conditions. Differ. Equ. 50(2), 213-219 (2014) (translated from Differ. Uravn. 50(2), 210-216 (2014))

20. Gasymov, MG: On the theory of polynomial operator pencils. Sov. Math. Dokl. 12, 1143-1147 (1972) (translated from Dokl. Akad. Nauk SSSR 199(4), 747-750 (1971))

21. Gasymov, MG: The multiple completeness of part of the eigen- and associated vectors of polynomial operator bundles. Izv. Akad. Nauk Arm. SSR, Ser. Mat. 6(2-3), 131-147 (1971) (in Russian)

22. Gasymov, MG: The solvability of boundary value problems for a class of operator-differential equations. Dokl. Akad. Nauk SSSR 235(3), 505-508 (1977) (in Russian)

23. Mirzoev, SS: Conditions for the well-defined solvability of boundary-value problems for operator differential equations. Sov. Math. Dokl. 28, 629-632 (1983) (translated from Dokl. Akad. Nauk SSSR 273(2), $292-295$ (1983))

24. Mirzoev, SS: Multiple completeness of root vectors of polynomial operator pencils corresponding to boundary-value problems on the semiaxis. Funct. Anal. Appl. 17(2), 151-153 (1983) (translated from Funkc. Anal. Prilozh. 17(2), 84-85 (1983))

25. Mirzoev, SS: Correct solvability of boundary value problems for even-order operator-differential equations. In: Spectral Theory of Differential Operators, pp. 85-91. Azerbaidzhan. Gos. Univ., Baku (1984) (in Russian)

26. Shkalikov, AA: Elliptic equations in a Hilbert space and related spectral problems. J. Sov. Math. 51(4), $2399-2467$ (1990) (translated from Tr. Semin. Petrovsk. 14, 140-224 (1989)) 
27. Yakubov, S, Yakubov, Y: Differential-Operator Equations: Ordinary and Partial Differential Equations. Chapman Hall/CRC Monogr. Surv. Pure Appl. Math., vol. 103. Chapman \& Hall, Boca Raton (2000)

28. Aliev, AR: Boundary-value problems for a class of operator differential equations of high order with variable coefficients. Math. Notes 74(5-6), 761-771 (2003) (translated from Mat. Zametki 74(6), 803-814 (2003))

29. Aliev, AR: On the boundary value problem for a class of operator-differential equations of odd order with variable coefficients. Dokl. Math. 78(1), 497-499 (2008) (translated from Dokl. Akad. Nauk 421(2), 151-153 (2008))

30. Aliev, AR, Gasymov, AA: On the correct solvability of the boundary-value problem for one class operator-differential equations of the fourth order with complex characteristics. Bound. Value Probl. 2009, 710386 (2009)

31. Aliev, AR, Mirzoev, SS: On boundary value problem solvability theory for a class of high-order operator-differential equations. Funct. Anal. Appl. 44(3), 209-211 (2010) (translated from Funkc. Anal. Prilozh. 44(3), 63-65 (2010))

32. Aliev, AR: On a boundary-value problem for one class of differential equations of the fourth order with operator coefficients. Azerb. J. Math. 1(1), 145-156 (2011)

33. Aliev, AR, Mohamed, AS: On the well-posedness of a boundary value problem for a class of fourth-order operator-differential equations. Differ. Equ. 48(4), 596-598 (2012) (translated from Differ. Uravn. 48(4), 587-589 (2012))

34. Aliev, AR: On the solvability of a fourth-order operator-differential equation with multiple characteristic. Ukr. Math. J. 66(5), 781-791 (2014) (translated from Ukr. Mat. Zh. 66(5), 699-707 (2014))

35. Lions, JL, Magenes, E: Non-homogeneous Boundary Value Problems and Applications. Dunod, Paris (1968) (Later editions: Mir, Moscow (1971), Springer, Berlin (1972))

36. Mirzoyev, SS: On the norms of operators of intermediate derivatives. Trans. Acad. Sci. Azerb. Ser. Phys.-Tech. Math. Sci. 23(1), 157-164 (2003)

\section{Submit your manuscript to a SpringerOpen ${ }^{\ominus}$ journal and benefit from:}

- Convenient online submission

- Rigorous peer review

Immediate publication on acceptance

- Open access: articles freely available online

- High visibility within the field

- Retaining the copyright to your article 